\title{
Corticoides tópicos como alternativa terapéutica en la dermatosis ampollosa por inmunoglobulina A lineal de la infancia. Caso clínico
}

\author{
Topical corticosteroids as a therapeutic alternative in linear immunoglobulin A \\ bullous dermatosis in childhood. Case report
}

\author{
Dr. Francisco José Gil Sáenza , Lic. Gabriel Durán Urdániza , Lic. Marta Fernández Galar \\ Lic. Juan Gimeno Ballester ${ }^{a}$, Lic. Ana Herrero Varas ${ }^{a}$ y Lic. Rosa Garcés Bordege ${ }^{a}$
}

\begin{abstract}
RESUMEN
La dermatosis por inmunoglobulina A lineal de la infancia es un trastorno autoinmunitario poco frecuente. Su etiología es desconocida, aunque se ha relacionado con fármacos, infecciones, enfermedades inmunológicas y procesos linfoproliferativos.

Presentamos el caso de una niña de 6 años que consultaba por lesiones ampollosas periorales, sin otra sintomatología. Se pautó un tratamiento con mupirocina tópica primero y luego con metilprednisolona tópica, sin resolución del cuadro. Se realizó una biopsia cutánea, compatible con dermatosis por inmunoglobulina A lineal. No fue posible iniciar el tratamiento con dapsona por déficit parcial de glucosa-6-fosfato deshidrogenasa, por lo que se mantuvo el tratamiento tópico, con buena evolución de las lesiones.

La dermatosis por inmunoglobulina A lineal es una enfermedad poco frecuente, cuyo diagnóstico diferencial incluye otras enfermedades ampollosas. La anatomía patológica es esencial para el diagnóstico. Si no es posible el tratamiento con dapsona, los corticoides tópicos pueden ser una alternativa, tanto en monoterapia como asociados a otros tratamientos.

Palabras clave: dermatosis IgA lineal, corticoesteroides tópicos, dapsona, glucosa-6-fosfato deshidrogenasa.
\end{abstract}

\section{ABSTRACT}

Linear immunoglobulin A dermatosis of childhood is a rare autoimmune disorder. Its etiology remains unknown, although it has been linked to drugs, infections, immunological diseases and lymphoproliferative processes.

We report the case of a 6 year old girl who consulted for perioral bullous lesions without other symptoms. Neither treatment with mupirocin nor methylprednisolone therapy achieved remission of cutaneous lesions. Skin biopsy showed a linear immunoglobulin A dermatosis. It was not possible to start treatment with dapsone because of a partial glucose-6-

a. Servicio de Pediatría.

b. Servicio de Dermatología.

Hospital García Orcoyen. Estella. España.

Correspondencia:

Dr. Francisco J. Gil Sáenz: fj.gil.saenz@cfnavarra.es

Financiamiento: Ninguno.

Conflicto de intereses: Ninguno que declarar.

Recibido: 22-3-2016

Aceptado: 3-5-2016 phosphate dehydrogenase deficiency, so topical treatment was maintained with good evolution of lesions.

Linear immunoglobulin A dermatosis is a rare disease whose differential diagnosis includes otherbullous diseases. Pathology is essential for diagnosis. When treatment with dapsone is not possible, topical corticosteroids may be an alternative, either alone or associated with other treatments.

Key words: linear IgA dermatosis, topical corticosteroids, dapsone, glucose-6-phosphate dehydrogenase.

http:/ /dx.doi.org/10.5546/aap.2016.e440

\section{INTRODUCCIÓN}

La dermatosis por inmunoglobulina A lineal (DAL) de la infancia es un trastorno autoinmunitario poco frecuente, ${ }^{1}$ caracterizado por el depósito lineal de inmunoglobulina A (IgA) en la unión dermoepidérmica. ${ }^{2}$ La incidencia a nivel poblacional publicada en la literatura varía de 0,1 a 2,3 casos/millón, sin clara predilección de género o etnia. ${ }^{1}$ Existen dos picos de incidencia en cuanto a la edad: el primero es en la infancia, de los 6 meses a los 5 o 6 años, y el segundo es en la edad adulta, habitualmente, en mayores de 60 años. ${ }^{1}$

La fisiopatología es desconocida, y están implicadas, probablemente, tanto la respuesta inmune celular como la humoral. ${ }^{2}$ Las lesiones tisulares son el resultado de la respuesta inflamatoria local inducida por los anticuerpos y la liberación de enzimas proteolíticas por neutrófilos y otras células inflamatorias. ${ }^{1,2}$ Se ha puesto en relación esta enfermedad con la presencia de determinados haplotipos de antígeno leucocitario humano (human leukocyte antigen; HLA, por sus siglas en inglés) (B8, Cw7, DR3, y DQ2) y con el factor de necrosis tumoral tipo 2 (tumor necrosis factor-2; TNF-2, por sus siglas en inglés). ${ }^{3}$

Los factores predisponentes o desencadenantes son muy variados, aunque lo más frecuente es que sea idiopática o inducida por fármacos. ${ }^{2} \mathrm{El}$ fármaco que con más frecuencia se ha asociado ha sido la vancomicina, aunque también se ha descrito 
asociada a antiinflamatorios no esteroideos (AINES), otros antibióticos, inhibidores de la enzima convertidora de angiotensina (IECAS) y otros fármacos. ${ }^{4-7}$ Otras enfermedades asociadas a la DAL son las oncológicas (sobre todo, neoplasias linfoproliferativas) y las autoinmunes (colitis ulcerosa y lupus eritematoso sistémico). ${ }^{1,8-10}$

Clínicamente, este cuadro se caracteriza por la aparición de lesiones vesiculosas, que pueden adoptar forma anular, lo que da lugar a las características lesiones en collar de perlas. ${ }^{2}$ Las lesiones también pueden afectar a mucosas (predominantemente, a la mucosa oral y conjuntival $)^{1}$ y comprenden, sobre todo, lesiones ulcerativas o erosiones. ${ }^{2}$ Aunque la afectación de mucosas es muy variable, parece ser más frecuente en adultos que en niños. ${ }^{11,12}$ Dadas las características clínicas, el diagnóstico diferencial se plantea, fundamentalmente, con otras enfermedades ampollosas, como la dermatitis herpetiforme, el impétigo bulloso o algunas formas de pénfigo y lupus. ${ }^{2}$

El tratamiento de elección es la dapsona (antibiótico del grupo de las sulfonas), aunque también existen otros tratamientos alternativos que pueden ser utilizados en caso de ineficacia o contraindicación. ${ }^{1,2}$

\section{CASO CLÍNICO}

Presentamos el caso clínico de una niña de 6 años que consultó por lesiones ampollosas periorales de 3 días de evolución. La paciente se encontraba afebril, había presentado algún vómito aislado sin diarrea ni otros síntomas. No refería antecedentes personales de interés ni tampoco tratamientos farmacológicos previos. $\mathrm{Al}$ momento de la exploración física, se observaban lesiones ampollosas de contenido claro de unos $0,2 \mathrm{~cm}$ de diámetro en la región facial, y el resto de la exploración era normal. Ante la sospecha de impétigo ampolloso, se recogió un cultivo de las lesiones y se inició un tratamiento con mupirocina tópica durante 10 días; el resultado del cultivo fue negativo.

Consultó nuevamente cuatro semanas después por la aparición de otras lesiones ampollosas. También refería prurito en el cuero cabelludo previo a la aparición de las lesiones. En la exploración física, se observaban lesiones vesículo-ampollosas tensas en la zona supraciliar, malar y glútea (Figura 1), con alguna pequeña lesión cicatricial residual. En la región cervical posterior y la región occipital, presentaba eritema descamativo con signos de rascado. Se solicitaron estudios analíticos, que incluyeron protoporfirinas y porfirinas en orina de 24 horas, que fueron normales. Se inició un tratamiento con metilprednisolona tópica $0,1 \%$ cada 12 horas en las lesiones cutáneas y clobetasol en champú para las lesiones en el cuero cabelludo.

Diez días más tarde, volvió a consultar por la aparición de lesiones anulares eritematosas discretamente induradas en la espalda, los codos y el abdomen (Figura 2). También

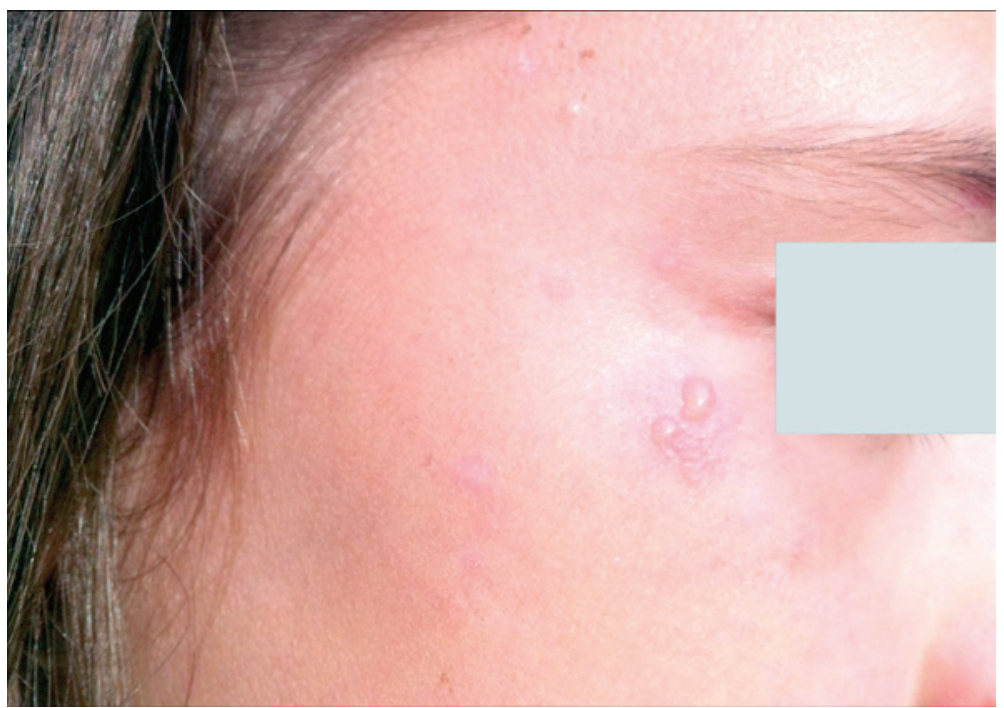


presentaba lesiones costrosas residuales de vesículas previas en la cara. Ante la sospecha clínica de enfermedad sistémica (lupus eritematoso, granuloma anular), se realizó una biopsia cutánea. El análisis anatomopatológico identificaba vesículas subepidérmicas con edema e infiltrados inflamatorios constituidos predominantemente por neutrófilos, y se observó, en la inmunofluorescencia directa, una positividad lineal con IgA en la zona de la membrana basal de la epidermis, todo ello compatible con DAL.

En ese momento, se planteó iniciar un tratamiento con dapsona. Se realizó, previamente, un análisis de sangre con determinación de glucosa-6-fosfato deshidrogenasa (G6PDH). Este fue compatible con déficit parcial de G6PDH, por lo que se decidió no iniciar el tratamiento con dapsona y se mantuvo el tratamiento con metilprednisolona tópica.

Un mes después, acudió a revisión, habiendo evolucionado muy favorablemente, con desaparición de las lesiones y sin aparición de otros elementos. Solo había quedado algún elemento residual hipopigmentado en la región facial. En la actualidad, lleva más de un año en remisión sin haberse registrado nuevos brotes.

\section{DISCUSIÓN}

La DAL es una enfermedad muy poco frecuente. ${ }^{1}$ El diagnóstico diferencial incluye la dermatitis herpetiforme, impétigo bulloso, pénfigo bulloso, lupus eritematoso bulloso y otras enfermedades ampollosas. ${ }^{2}$ La anatomía patológica es una herramienta esencial para el diagnóstico; la demostración de depósitos lineales de $\operatorname{Ig} \mathrm{A}$ en la membrana basal por inmunofluorescencia directa es el estándar de oro para el diagnóstico. ${ }^{13}$

Dado que, en bastantes ocasiones, esta entidad es secundaria a fármacos, es esencial retirarlos para el manejo de la enfermedad. ${ }^{1,2} \mathrm{Al}$ margen de esto, el tratamiento farmacológico de primera línea en esta patología es la dapsona. ${ }^{1,2,4}$ Aunque este es un tratamiento eficaz, rápido y bien tolerado por la mayoría de los pacientes, debe ser administrado con precaución por sus posibles efectos adversos graves, tales como hemólisis, metahemoglobinemia, agranulocitosis, reacciones de hipersensibilidad, neuropatía periférica, hepatitis y nefritis. ${ }^{2,4}$ Además, antes de iniciar su uso, todos los pacientes deberían ser testados para el déficit de G6PDH, puesto que esta medicación puede originar cuadros graves de hemólisis en los pacientes con deficiencia de esta enzima. ${ }^{1,2}$ En los

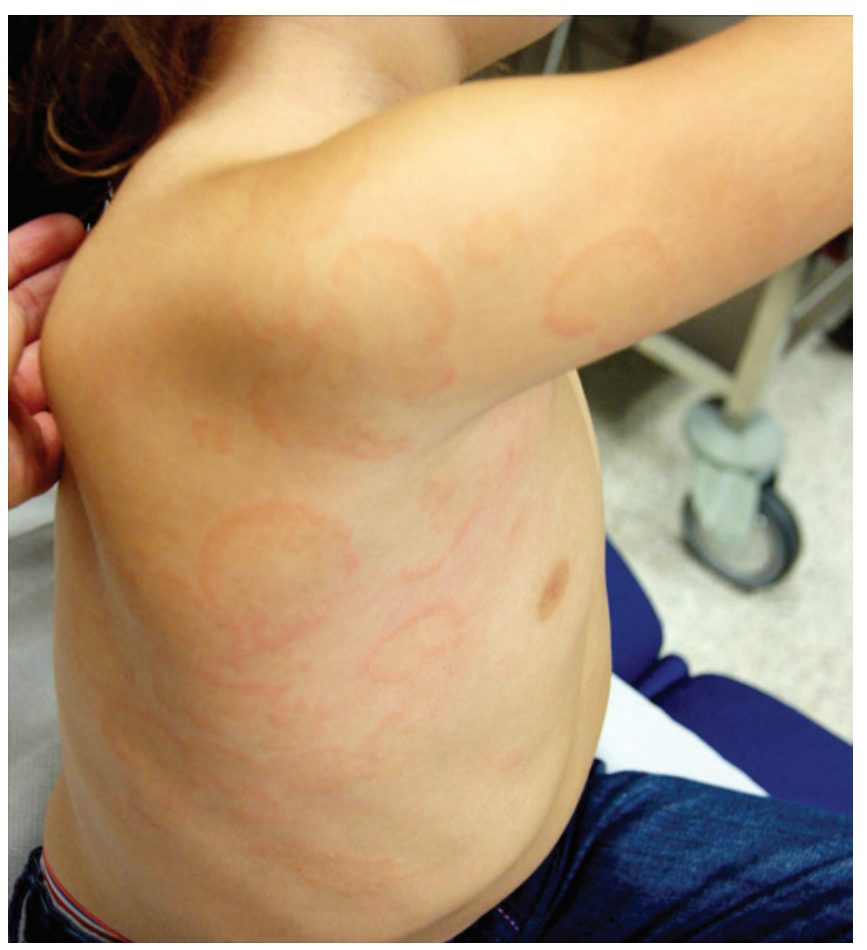


casos en los que la dapsona no puede ser utilizada, existen, como tratamientos de segunda línea, las sulfonamidas, ${ }^{1,2,4}$ que serían mejor toleradas, aunque tendrían un perfil de efectos adversos similar a la dapsona. ${ }^{1,2}$ Este tipo de medicamentos también fueron, en el pasado, contraindicados para pacientes con déficit de G6PDH, aunque, hoy en día, se piensa que su uso puede ser seguro en estos pacientes en las dosis habituales. ${ }^{14}$ Otro medicamento de segunda línea es la colchicina, ${ }^{1,2,4}$ que se ha utilizado tanto en asociación como en monoterapia, con mejores resultados en niños que en adultos; ${ }^{2}$ además, es una medicación segura en pacientes con déficit de G6PDH. ${ }^{14}$

En los casos refractarios, también pueden ser útiles agentes inmunosupresores (como corticoides sistémicos, micofenolato mofetil o ciclosporina), ${ }^{1,2,4}$ inmunoglobulinas intravenosas e inmunoabsorción ${ }^{1,2}$ y antibióticos sistémicos (tetraciclinas, macrólidos, trimetroprínsulfametoxazol), ${ }^{1,2,4}$ todos ellos solos o en asociación.

Al margen de las terapias sistémicas, algunos autores consideran que el tratamiento con corticoides tópicos puede ser suficiente en monoterapia para controlar casos leves o de enfermedad localizada. ${ }^{1,2,4,15}$ Sin embargo, en la mayoría de los casos, son usados como terapia adyuvante al inicio del tratamiento, y se utilizan corticoides de alta potencia una o dos veces al día hasta 2 semanas. ${ }^{2}$

En nuestro caso, dada la imposibilidad de iniciar el tratamiento con dapsona, se optó por continuar con un tratamiento tópico, que se mantuvo aplicándose a los sucesivos brotes durante 8 semanas hasta conseguir el control de la enfermedad. Aunque la evolución, en el inicio, no fue hacia una rápida mejoría y se produjo una extensión de las lesiones, finalmente, el proceso se pudo controlar manteniendo el tratamiento tópico durante un período prolongado. Los corticoides tópicos, sobre todo los de alta potencia, pueden tener efectos secundarios importantes y riesgo de atrofia cutánea, ${ }^{2}$ por lo que no se recomienda su uso de manera prolongada. Sin embargo, en este caso, la enfermedad evolucionaba en brotes que afectaban a distintas partes del cuerpo. El uso de esta medicación en distintas zonas corporales de forma sucesiva condujo al control de la enfermedad sin la aparición de efectos adversos, lo que evitó tener que utilizar otros tratamientos sistémicos con una potencialidad tóxica superior.

El pronóstico de esta enfermedad es hacia un curso crónico de 3 a 6 años antes de la remisión. ${ }^{4}$
En caso de recidiva, se recomienda reiniciar la terapia sistémica y mantenerla durante semanas o meses tras la remisión. ${ }^{1}$

Aunque, actualmente, no se puede establecer una relación causal con enfermedades autoinmunes o neoplásicas por la baja incidencia de esta enfermedad y el alto porcentaje de casos idiopáticos, ${ }^{1}$ se necesitan más datos para saber si la asociación encontrada en algunos casos es causal o no. ${ }^{1}$ De cualquier manera, teniendo en cuenta estas asociaciones y el curso crónico de la enfermedad, parece recomendable realizar un seguimiento de estos pacientes durante años, al menos, hasta la adolescencia en los casos infantiles.

\section{REFERENCIAS}

1. Fortuna G, Marinkovich MP. Linear immunoglobulin A bullous dermatosis. Clin Dermatol 2012;30(1):38-50.

2. Hall RP, Rao CL. Linear IgA bullous dermatosis [Internet]. Waltham: UpToDate; 2016. [Acceso: 2 de febrero de 2016]. Disponible en: http://www.uptodate.com/contents / linear-iga-bullous-dermatosis?source=search_result\& search=linear+iga+bullous+dermatosis selectedTitle=1 22.

3. Collier PM, Wojnarowska F, Welsh K, McGuire W, et al. Adult linear IgA disease and chronic bullous disease of childhood: the association with human lymphocyte antigens Cw7, B8, DR3 and tumour necrosis factor influences disease expression. Br J Dermatol 1999;141(5):867-75.

4. Ng SY, Venning VV. Management of linear IgA disease. Dermatol Clin 2011;29(4):629-30.

5. Bouldin MB, Clowers-Webb HE, Davis JL, McEvoy MT, et al. Naproxen-associated linear IgA bullous dermatosis: case report and review. Mayo Clin Proc 2000;75(9):967-70.

6. Ho JC, Ng PL, Tan SH, Giam YC. Childhood linear IgA bullous disease triggered by amoxicillin-clavulanic acid. Pediatr Dermatol 2007;24(5):E40-3.

7. Friedman IS, Rudikoff D, Phelps RG, Sapadin AN. Captopril-triggered linear IgA bullous dermatosis. Int J Dermatol 1998;37(8):608-12.

8. Godfrey K, Wojnarowska F, Leonard J. Linear IgA disease of adults: association with lymphoproliferative malignancy and possible role of other triggering factors. $\mathrm{Br} J$ Dermatol 1990;123(4):447-52.

9. Paige DG, Leonard JN, Wojnarowska F, Fry L. Linear IgA disease and ulcerative colitis. Br J Dermatol 1997;136(5): 779-82.

10. Tobón GJ, Toro CE, BravoJC, Cañas CA. Linear IgA bullous dermatosis associated with systemic lupus erythematosus: a case report. Clin Rheumatol 2008;27(3):391-3.

11. Wojnarowska F, Marsden RA, Bhogal B, Black MM. Chronic bullous disease of childhood, childhood cicatricial pemphigoid, and linear IgA disease of adults. A comparative study demonstrating clinical and immunopathologic overlap. J Am Acad Dermatol 1988;19(5 Pt 1):792-805.

12. Horiguchi $Y$, Ikoma A, Sakai R, Masatsugu A, et al. Linear IgA dermatosis: report of an infantile case and analysis of 213 cases in Japan. J Dermatol 2008;35(11):737-43.

13. GuideSV,Marinkovich MP. Linear IgA bullous dermatosis. Clin Dermatol 2001;19(6):719-27.

14. Youngster I, Arcavi L, Schechmaster R, Akayzen Y, et al. Medications and glucose-6-phosphate dehydrogenase deficiency: an evidence-based review. Drug Saf 2010;33(9):713-26.

15. Culton DA, Diaz LA. Treatment of subepidermal immunobullous diseases. Clin Dermatol 2012;30(1):95-102. 